\title{
El trabajo colegiado en la escuela normal
}

\author{
Efrén Viramontes Anaya \\ Escuela Normal Rural Ricardo Flores Magón \\ efren8000@hotmail.com
}

\section{Resumen}

El objeto de estudio de esta investigación es el trabajo colegiado de un grupo de académicos de la Escuela Normal Rural "Ricardo Flores Magón”, pertenecientes al 7ํ y 8oㅗㄹ semestres de la Licenciatura en Educación Primaria. El proceso indagatorio se realizó durante los ciclos escolares 2003-2004 y 2004-2005, abordando dos propósitos principales: uno, sobre la percepción que tienen los docentes de la academia con respecto a sus actividades colegiadas; $y$, el segundo, en relación al papel que tiene el trabajo colegiado en la escuela normal. La metodología emplea da en esta investigación es la etnográfica, el modo epistémico es interpretativo descriptivo y las técnicas empleadas son observación participante, análisis de documentos y entrevista de los integrantes del grupo focal. Los resultados se expusieron en tres partes: lo relacionado con las condiciones generales del trabajo colegiado en la escuela normal, la descripción y análisis del trabajo colaborativo al interior de la academia y el impacto del trabajo colegiado en la formación de las estudiantes y el desarrollo institucional.

\section{Palabras clave \\ Trabajo colegiado, trabajo académico, escuela normal.}

\section{Introducción}

En la actualidad son pocas las investigaciones que se han realizado sobre esta temática, Espinosa (2004), representa en la actualidad una de las aportaciones más estructuradas en este tópico. Presenta, además, una relación sobre las investigaciones existentes relacionadas con el trabajo colegiado, señalando cuáles estudios son y de dónde proviene cada uno de ellos:

... de la Nacional de Educadoras, el informe del sabático sobre la puesta en marcha de la Licenciatura en Educación Preescolar y su seguimiento por un año, 1999-2000, donde se analiza, entre otras cosas, el trabajo colegiado. De la Veracruzana tenemos dos artículos publicados en la revista Didacta, el primero es el previo al plan $97 \mathrm{y}$, por lo mismo, muestra una reflexión sobre el trabajo de las academias del plan 84; mientras que el segundo se refiere, específicamente, al funcionamiento de las academia con el nuevo plan, en concreto al $4^{\circ}$ semestre de la Licenciatura en Educación Primaria. También hay un tercer documento, una investigación más amplia que las anteriores, y trata del seguimiento y de la evaluación de de Educación Primaria en la propia escuela normal, además incluye 
una reflexión acerca de las academias o colegio (p. 9).

\section{Problemática}

El principio del planteamiento coincide con lo que ya ha sido observado y estudiado por Czarny (2003) en una investigación sobre el trabajo colegiado de las escuelas normales en la que señala que las principales dificultades que percibió fueron las siguientes:

- La falta de participación y la irregular asistencia de todos los profesores y autoridades involucradas.

- La discontinuidad en las reuniones y de los temas que en cada una de ellas de abordan.

- Problemas en las relaciones interpersonales entre los participantes.

- La carencia de tiempo para tratar todos los temas que se requiere, así como la falta de seguimiento a los acuerdos que se toman (p. 33).

A estos señalamientos se pueden agregar otros que de manera particular se tienen en la Escuela Normal Rural "Ricardo Flores Magón" como la falta de un espacio temporal común de cada uno de los integrantes del cuerpo docente, la falta de compromiso, la diversidad de posturas políticas $\mathrm{y}$, principalmente la falta de compromiso institucional de algunos maestros. Esta percepción de problemática es la que se tenía al momento de iniciar formalmente con el proceso de investigación de este objeto de estudio. Se pensó que esas situaciones creaban un ambiente de trabajo que hacía más difícil el desarrollo curricular de las Licenciaturas en Educación Primaria y Preescolar.

Ejes centrales del objeto de estudio.

- ¿Cómo perciben los integrantes de la academia $7^{\circ}$ y $8^{\circ}$ o semestres de la Licenciatura en Educación Primaria el proceso del trabajo colegiado que se realiza en la Escuela Normal Rural "Ricardo Flores Magón?

- ¿Cuál es el papel del trabajo colegiado en la formación docente, tomando como referencia las exigencias del plan de estudios vigente 1997 de la Licenciatura en Educación Primaria?

\section{Cuestiones específicas del objeto de estudio.}

Los siguientes cuestionamientos que se presentan, tienen una relación directa con los objetivos que se incluyen en esta investigación $\mathrm{y}$ han sido planteados de tal forma que en su conjunto respondan a los dos ejes centrales de este estudio.

Además, estas cuestiones específicas me aportaron una directriz muy clara para plantear categorías de análisis que respondan directamente a sus planeamientos, toda vez que representan puntos nodales del objeto de estudio de la investigación.

- ¿Cómo se realiza el trabajo colegiado en la escuela normal?

- ¿Quiénes participan y cómo lo hacen?

- ¿Qué avances se perciben en el mejoramiento de las prácticas docentes de los maestros que participan en las academias?

- ¿Qué impactos se perciben en las estudiantes como resultado del trabajo colegiado?

- ¿Qué problemas se perciben como obstaculizadores del trabajo colegiado?

- ¿Qué tipo de participación tienen los maestros de un grupo colegiado?

- ¿Cómo se manifiesta la cooperación en el desarrollo de las actividades del colegiado?

\section{Objetivos de la investigación}

Los objetivos de esta investigación se derivan de los ejes centrales y las cuestiones específicas de este mismo trabajo y se 
plantean como el conjunto de expectativas que se tienen para cumplir mediante el proceso investigativo.

1. Caracterizar el trabajo colegiado que se desarrollan al interior de la academia de maestros que fue motivo de estudio.

2. Conocer el impacto que tienen lo procesos del trabajo colegiado en el mejoramiento de las prácticas profesionales y en el aprendizaje colectivo de los maestros.

3. Valorar los tipos de participación que tienen los integrantes de un colegiado.

4. Describir las situaciones problemáticas que se viven en el trabajo colegiado.

5. Tener los elementos para elaborar un proyecto de desarrollo e impulso al trabajo colegiado de la escuela normal donde se realizó la investigación.

\section{Referentes teóricos}

Los respaldos teóricos principales que se utilizaron en la investigación son las siguientes:

- Enfoque participativo de la gestión escolar y el trabajo colaborativo (Antúnez 1998 y Ball 1994)

- Los estilos del director de la escuela (Antúnez 1998 y Ball 1994)

- Teoría de los grupos operativos (Zarzar Charur, 1980).

- Liderazgo (Schlmelkes, 2001)

- Participación (Viñas y Doménech, 1994)

- Planes de estudio y documentos oficiales de la SEP

\section{Metodología de la investigación}

Los sujetos que fueron parte de esta investigación son todos los docentes de la Escuela Normal "Rural Ricardo Flores Magón”, considerados como parte de las academias establecidas por la estructura organizativa de la escuela. Los docentes fueron la unidad mínima considerada como sujeto de investigación.

El trabajo colegiado en la escuela normal
Los criterios principales de la selección (diferente a muestra) de los sujetos se realizó conforme a lo establecido por Goetz y LeCompte (1988), específicamente en lo relacionado con la conformación natural de los grupos o unidades iniciales de análisis, considerados como fuentes principales de información: las academias; también por el hecho de que el propósito de esta investigación no es la generalización por inferencia.

Los escenarios en los que se realizó la investigación, generalmente se redujo a los espacios que cada academia tenga determinados, aunque por lo general se desarrollan en la sala de maestros, pero no siempre se hacen ahí.

La presente investigación se ubica en el enfoque cualitativo, toda vez que se desarrollará en un proceso en que los datos serán tratados de manera holística; individual, más que general. En lo que corresponde al paradigma de investigación en el que se sitúa este trabajo es interpretativo.

La ubicación en el paradigma interpretativo tiene la justificación de que, por lo menos en esta primer etapa, las pretensiones son sólo las de describir, comprender e interpretar lo que sucede en las academias; por consiguiente, quedaría lejos de cualquier otro propósito característico de los dos paradigmas restantes.

El tipo de investigación al que se está apuntando hasta el momento, es a la etnográfica, ya que coincide con la idea general que se tiene de la investigación y de sus posibles productos, así como con los objetivos planteados en la misma.

La entrada al "campo" (en términos de la etnografía) no representó ningún problema. En lo general el trabajo de campo se realizó permanentemente en todas las reuniones de la academia durante los ciclos escolares 20032004 y 2004-2005.

La recogida de datos que se utilizó como insumo de esta investigación, se realizó 
a través de las siguientes técnicas e instrumentos:

Se realizaron entrevistas a los docentes de la academia de $7^{\circ}$ y $8^{\circ}$ semestre de la Licenciatura en educación Primaria. Las entrevistas se desarrollarán respondiendo al enfoque cualitativo, con preguntas abiertas y no estructuradas.

Otra técnica de acopio de información, utilizado fue la que se denomina "observación participante", a través de la cual se llevó a cabo la recolección de datos más directos que se produjeron en los escenarios donde se realizó el trabajo colegiado.

Registro de diario de campo, a modo de relatoría, de lo que sucedió al interior de las reuniones de academia.

Registro de academias, misma que se realizó por uno de los miembros de ellas, generalmente fue rotativo: esta actividad se realizó en un diario (cuaderno grande con pastas gruesas), en donde quedó asentado lo que se trató en cada una de las reuniones, mediante un estilo muy variado, desde registros muy detallados hasta escritos totalmente esquemáticos y parcos en la descripción.

El análisis de la información se realizó con base en las actividades de "comparación, contrastación, agregación y ordenación" que propone Goetz y LeCompte (1988).

Para realizar el análisis, se utilizaron o construyeron categorías que permitieron sistematizar la información, organizarla y utilizarla de tal forma que aportara ideas o estructura que dan sentido a los descubrimientos, interpretaciones $y$ descripciones que se realizan.

Para esta investigación se pueden anticipar desde este momento una serie de categorías iniciales que, fueron necesarias para realizar el trabajo de análisis anteriormente indicado. Las categorías de análisis de la investigación son las siguientes:

- Estilos de trabajo colegiado

- Productos del trabajo colegiado

- Temáticas de las agendas del trabajo colegiado

- Características que promueven buenos productos y procesos del trabajo colegiado

- Problemas que afectan un provechoso trabajo colegiado

- Acciones administrativas en torno al trabajo colegiado

- Respuesta general de los docentes hacia la participación colectiva

- Impactos del trabajo colegiado en la formación docente y perfil de egreso de las alumnas

- Impactos del trabajo colegiado en el aprendizaje de los docentes y el mejoramiento de sus prácticas docentes.

\section{Resultados de la investigación: primera categorización del proceso del trabajo colegiado}

Esta primera parte de los resultados contiene un sistema de categorización que se integra y explica a través de la figura 1.

Lo recuadros obscurecidos, muestran la columna principal de categorías de análisis, de las cuales emergen un sistema de subcategorías que intentan dar una interpretación general y específica a la vez de los qué y cómo se hace el trabajo colegiado en la academia de 7으 y $8^{\circ}$ semestres de la Escuela Normal Rural "Ricardo Flores Magón”. 


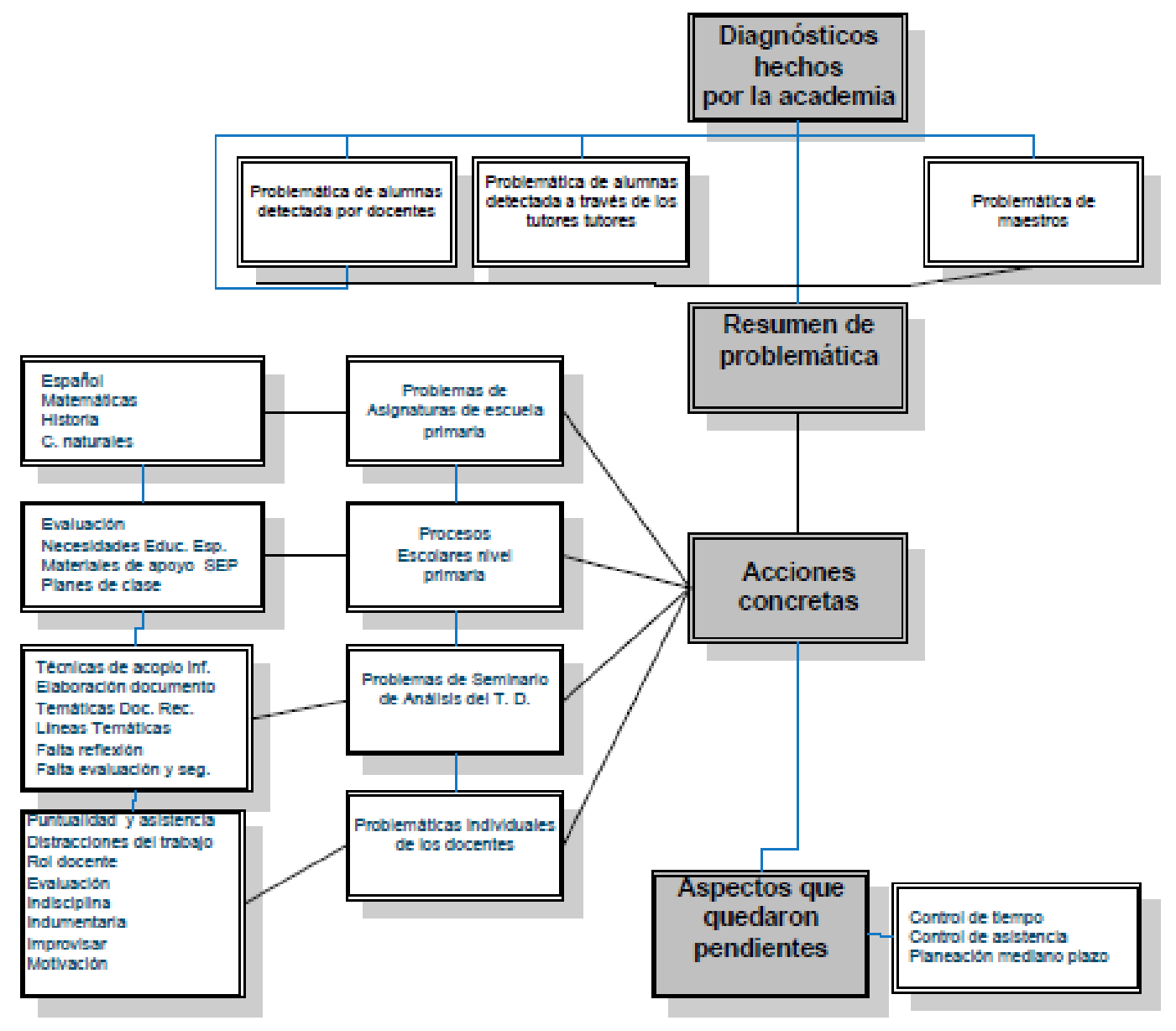

Figura 1. Acciones del Grupo Colegiado

Interpretación del proceso, papel e impacto del trabajo colegiado en la escuela normal

Con el análisis de las categorías que se presentan hasta aquí, combinado con la experiencia y la observación como docente e investigador en torno al objeto de estudio, se dio sentido a un modelo o constructo teórico que ayuda a considerar de manera holística e integral la interpretación del papel del trabajo colegiado y el impacto que tiene en la escuela normal.

Esto se ilustra en la figura 2.

Para hacer esta interpretación global, se consideró hacerlo a través de cuatro partes fundamentales: la primera, contiene las categorías que hacen alusión a las condiciones generales en las que se desarrolla la academia de $7^{\circ}$ y $8^{\circ}$ semestres. La segunda, se refiere al análisis de los procesos internos de interacción personal que se vive para la realización del trabajo colaborativo. La tercera, expone todo lo relacionado con las actividades, papel y productos del trabajo colegiado, en las que se puntualizan los elementos que se generan por el colegio. En la cuarta y última se incluyen las categorías que se relacionan con los productos de la actividad de la academia y los ámbitos en que se presenta. 


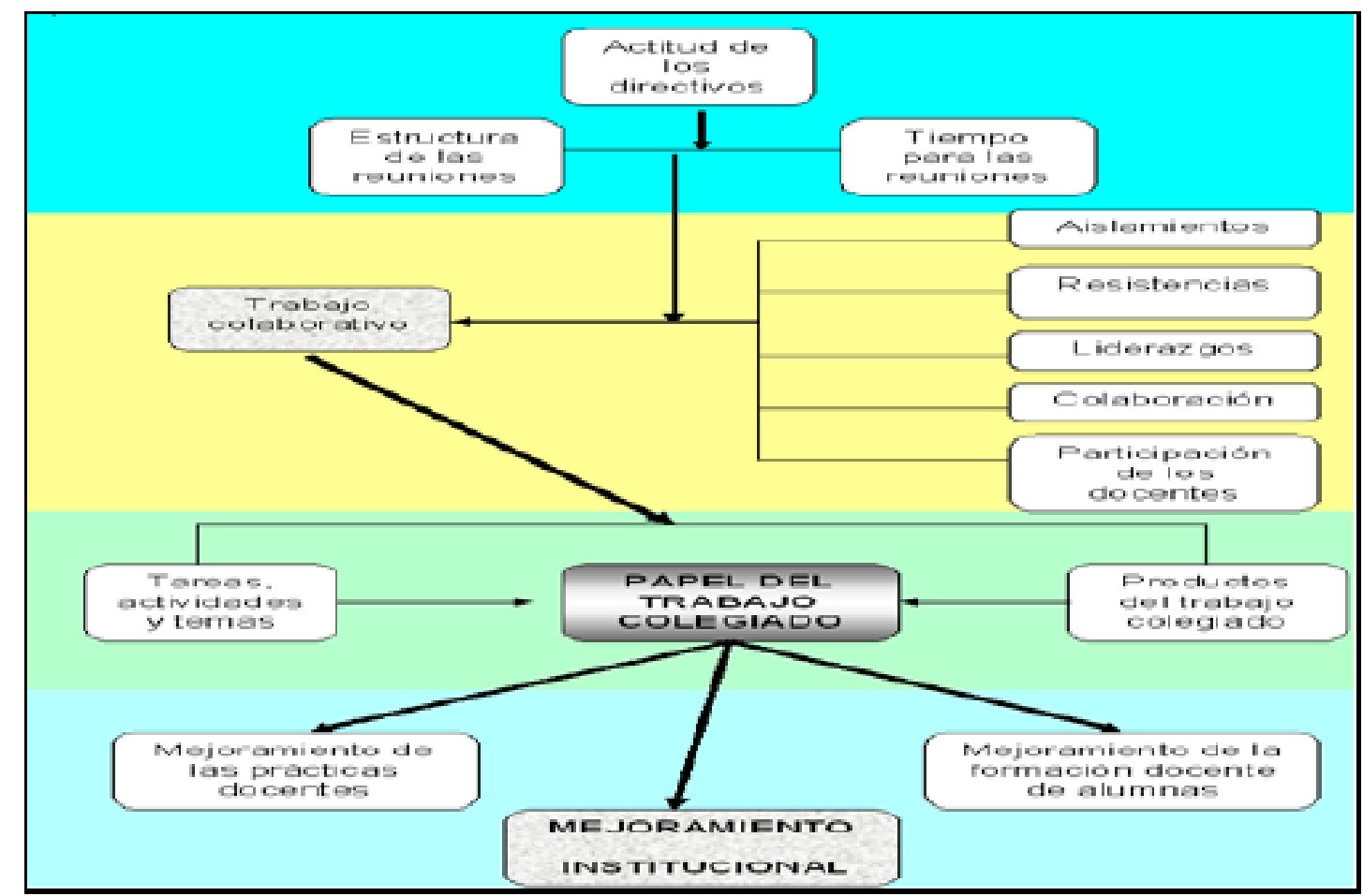

Figura 2. Esquema interpretativo de las condiciones, proceso, papel e impacto del trabajo colwgiado.

\section{Conclusiones}

En lo general, como una parte importante de estas conclusiones, se puede afirmar, que existen factores muy variados que caracterizan y condicionan la realización del trabajo colegiado, mismos que van desde que los directivos tengan una actitud de apoyo, promoción y valoración de las actividades colectivas, hasta tener las condiciones, organización, estructura, tiempo y elementos que estén dispuestos a iniciar procesos colegiados; todo esto bajo la sensibilización o creación de un clima de necesidades concretas en la que los integrantes le encuentren sentido a lo que tienen o deben hacer en las sesiones de trabajo.

Respuestas a las preguntas principales de esta investigación:

1) Por un lado se plantea que la manera cómo los docentes de la academia de $7^{\circ}$ y $8^{\circ}$ semestres perciben el trabajo colegiado es ampliamente limitada al no ser conscientes de lo que realmente se realiza el colectivo, principalmente en lo relacionado con las actividades y funciones de este grupo;

2) Por otro lado, se percibió de manera más contundente, que el papel que tiene el trabajo colegiado en la escuela normal, impacta decididamente en el mejoramiento de la formación de las estudiantes normalistas; en el mejoramiento de las prácticas de los docentes involucrados en la academia; $y$, finalmente, en el mejoramiento institucional y de la gestión escolar en lo general.

También se obtuvieron conclusiones sobre las siguientes categorías:

- La actitud de los directivos

- La estructura y formato de las reuniones de academias

- Los procesos interactivos que se dan al interior de las reuniones

- La cooperación o colaboración entre los integrantes del colegio

- Los procesos de colaboración 
- El liderazgo

- Aislamiento

- Resistencias

- Tipos de participaciones

- Acciones, productos y papel del trabajo colegiado

- La percepción que los docentes de la academia de $7^{0}$ y $8^{\circ}$ semestre tienen con respecto a las actividades y funciones que realizan en el colegio

- Vinculación con el entorno

- Impacto que tiene el trabajo colegiado en las alumnas, en las prácticas de los maestros y en el mejoramiento general de la institución

Finalmente, es pertinente cerrar este apartado con dos recomendaciones específicas: la primera, dirigida a los maestros de las escuelas normales, para que se animen a participar en los procesos colegiados, tomando en cuenta que pueden mejorar sus prácticas y la formación de sus alumnas, respondiendo al compromiso moral $\mathrm{y}$ profesional que tenemos los docentes para con nuestros estudiantes; y, la segunda, enfocada hacia los directivos de las escuelas, para que observen las condiciones en que se desarrolla el trabajo colegiado, la forma en que valoran el hecho de que los maestros se organicen y propongan acciones pedagógicas, organizacionales, administrativas, de vinculación, político-laborales y de convivencia social, sin temerle a los riesgos que pudiera tener, asumiendo una actitud menos egocéntrica, arriesgándose sin importar que en el proceso pueda ser aparentemente sacrificado status en la estructura vertical, en aras de un desarrollo institucional efectivo y genuino que involucre la comunidad normalista

\section{Referencias}

Antúnez, Serafín (1999), El trabajo en equipo como factor de calidad: el papel de los directivos escolares. Versión corregida de la conferencia dictada en Cancún, Quintana Roo, junio.

Alberto A. (1998). La federación educativa en México 1889-1994. SEP/COLMEX/ CIDE, México.

Ball, S. J.(1994). La micropolítica en la escuela: hacia una teoría de la organización escolar. Barcelona, Paidós.

Czarny, Gabriela. (2003) Las escuelas normales frente al cambio: Un estudio de seguimiento a la aplicación del Plan de estudios 1997. México: SEP.

Espinosa Carvajal, M. E. (2004) El trabajo colegiado: su funcionamiento, sus aportes y dificultades en tres escuelas normales México, SEP-SEByN.

García Alcaraz, M. G. (1997) Revista La tarea. Guadalajara, Méx. 1997 s/p.

Gobierno De Los Estados Unidos Mexicanos. Presidencia De La República. El Plan Nacional de Desarrollo 2001-2006. México: Poder ejecutivo federal

Goetz, J.P. y LeCompte, M. D. (1988). Etnografía y diseños cualitativos en investigación educativa. "Evaluación del diseño etnográfico". Madrid. Ediciones Morata, S.A.

Latapí S., P. (2002). ¿Cómo aprenden los maestros? Observatorio ciudadano de la educación. En: http://www.scielo.org.mx/scielo.php? script=sci_nlinks\&ref $=1008951 \&$ pid $=$ S01852698201000010000500036\&lng=es

Macías, H. (sin fecha). Comunidades de aprendizaje, modelo paradigmático. http://kino.tij.uia.mx/ humberto/co mun3.html

Pourtois, J.P. y Desmet, H. (1994). Las dos tradiciones científicas: en: Antología Básica: Construcción social del conocimiento y teorías de la educación. México, SEP-UPN.

Rodríguez G., J. y otros (1999). Metodología de la investigación cualitativa Segunda edición. Málaga, Aljibe. 
Schlmelkes, S. (2001). Calidad de la educación y la gestión escolar.

SEP (1997). Plan de estudios y programas de la Licenciatura en Educación Primaria 1997. La importancia del trabajo colegiado. México. SEP.

SEP-DGN (1998). Propuesta para el desarrollo del trabajo colegiado en las escuelas normales. México: SEP.

SEP-DGN (2003). El diagnóstico institucional en las escuelas normales. Orientaciones para su elaboración Serie: gestión institucional 3. México: SEP.

SEP-SEByN (2003). Datos básicos sobre la educación normal en México: en: Gaceta de la escuela normal. Mes de abril. México, SEP-SEByN.

SEP-SEByN (2003). El mejoramiento de la gestión institucional en las escuelas normales (Elementos para la reflexión y análisis) Serie: Gestión Institucional 2. México: SEP.

SEP-SEByN (2003). El mejoramiento de la gestión institucional en las escuelas normales (Elementos para la reflexión y análisis) Serie: Gestión Institucional 1. México: SEP.

SEP-SEByN (2004). Seguimiento y evaluación de las prácticas docentes: una estrategia para la reflexión y mejora de las escuelas normales. México: SEP.

SEP-SEByN. Normatividad académica. Propuesta para el desarrollo del trabajo colegiado en las escuelas normales que ofrecen la licenciatura en educación preescolar. México: SEP.

Torres, R.M. (2001). Comunidades de aprendizaje. Repensando lo educativo desde el desarrollo local y desde el aprendizaje. Barcelona Forum 2004.

Viñas C., J Y Doménech, F (1994) Toma de decisiones y la participación, en: Joaquín Garín Santillán y Pere Darder Vidal (coords.) Organizción de los centros educativos.spectos básicos, Barcelona, Praxis (Praxis Universidad),

Zarzar Charur, C. (1980). Grupos de aprendizaje. Nueva imagen. México. 